\title{
Effect of size on the luminescent efficiency of
}

\section{perovskite nanocrystals}

J. T. Griffiths ${ }^{1}$, F. Wisnivesky Rocca Rivarola ${ }^{1}$, N. J. L. K. Davis ${ }^{2}$, R. Ahumada-Lazo ${ }^{3}$, Juan Arturo Alanis Azuara ${ }^{3}$, P. Parkinson ${ }^{3}$, D. J. Binks ${ }^{3}$, W. Y. Fư ${ }^{4}$, F. De La Pena ${ }^{1}$, M. B. Price ${ }^{2}$, A. Howkin $^{5}$, I. Boyd ${ }^{5}$, C. J. Humphreys ${ }^{1,6}$, N. C. Greenham ${ }^{2}$, C. Ducati ${ }^{1}$

1. Department of Materials Science and Metallurgy, University of Cambridge, Charles Babbage Road, Cambridge, CB3 0FS, United Kingdom

2. Cavendish Laboratory, University of Cambridge, J. J. Thompson Avenue, Cambridge, CB3 0HE, United Kingdom

3. School of Physics and Astronomy and the Photon Science Institute, University of Manchester, Manchester, M13 9PL, United Kingdom

4. Department of Electrical and Electronic Engineering, University of Hong Kong, Pokfulam Road, Hong Kong

5. Experimental Techniques Centre, Brunel University, Uxbridge, UB8 7BQ, United Kingdom

6. School of Engineering and Materials Science, Queen Mary University of London, London, E1 4NS, United Kingdom

\section{Abstract}


Perovskite colloidal nanocrystals have emerged as important new optical materials, with tuneable light emission across the visible spectrum, narrow linewidths for high colour purity, and quantum efficiencies approaching unity. These materials can be solution processed in large volumes at low cost making them promising for optoelectronic devices.

The structure of nanocrystals influences the radiative and non-radiative recombination of carriers within them through trap states and Auger recombination. To optimise the emission properties it is vital to understand the relationship between the optical emission of individual nanocrystals and their structure, size, and composition of individual nanocrystals.

Here, we use nano-cathodoluminescence to relate the nanoscale optical emission of individual inorganic perovskite nanocrystals to their size. This approach reveals that larger nanocrystals exhibit brighter luminescence, indicating lower non-radiative losses compared to smaller nanocrystals. We also show nanoscale colour mixing with bright red and blue emission from individual $\mathrm{CsPbI}_{3}$ and $\mathrm{CsPbCl}_{3}$ nanocrystals respectively in mixed films.

The optical and structural characterisation serve as a powerful approach to the study of colloidal semiconductor nanocrystals that improves the fundamental understanding of quantum structures leading to improved optoelectronic devices.

The increasing demand for sustainable development has led to the search for more efficient optical materials for light emitting and harvesting devices, and driven the development of novel nanostructures. 
Nanocrystal emission properties can be precisely controlled by variations in their size and composition ${ }^{1,2}$. This gives rise to high colour purity over a wide spectral range with spatially localised emission, making them desirable for displays ${ }^{3}$. Remarkably high emission efficiencies often approaching unity have been shown and combined with their controllable emission wavelength makes them well-suited to lighting applications ${ }^{4}$, as well as promising gain media for low-threshold lasers ${ }^{5}$. Many nanocrystals structures have high absorption cross-sections making them suitable energy harvesting materials for the next-generation graded multi-junction solar cells ${ }^{6}$. These desirable properties combined with low cost synthesis makes them attractive for large area and low cost devices ${ }^{7}$. In particular, perovskite colloidal semiconductor nanocrystals have emerged recently as promising materials for these applications ${ }^{8,9}$.

The emission dynamics of luminescent materials are determined by the competition between different processes, such as trapping at surface states, radiative band to band transitions, and Auger recombination.

Trapping of carriers at surface states can lead to non-radiative recombination reducing the overall quantum efficiency. Carriers can also escape from the traps, returning to a band edge state ${ }^{10}$. The rate of trapping can vary substantially with the size of the nanocrystals as the surface to volume ratio changes. The trapping and trap-escape times depend sensitively on the nature and location of the surface state, varying by many orders of magnitude even for the same material ${ }^{11-13}$.

Auger recombination can reduce emission in nanocrystals as the energy released by electron-hole recombination is transferred non-radiatively to another carrier ${ }^{14}$. This therefore increases at higher excitation fluences when multiple excitons are created within a nanocrystal. It can also occur at low fluence when one of the carriers from a photo-generated electron-hole pair becomes trapped at the surface and the other survives within the nanocrystal volume until a subsequent photo-excitation event and so is available to enable Auger recombination; which is one of the causes of the well-known intermittent luminescence effect in nanocrystals, known as 'blinking' ${ }^{10}$. In nanocrystals, the Auger lifetime scales linearly with nanocrystal volume, independent of the bulk semiconductor material band structure ${ }^{15}$. When the nanocrystal size increases relative to the Bohr radius and enters the weak 
confinement regime, the Auger lifetime deviates from the 'universal volume scaling' and shows sublinear dependencies. ${ }^{16}$ As the size continues to increase, the Auger lifetime becomes dependent on the electronic structure of the bulk material. For perovskite nanocrystals with weak confinement the rate of Auger recombination has previously been shown to scale sub-linearly with volume ${ }^{16}$.

Understanding the relationship between the structure and size of individual nanocrystals and their recombination processes is essential to the development of high efficiency semiconductor materials for optoelectronic devices.

Optical spectroscopy techniques can yield information on carrier dynamics for ensembles of nanocrystals, but lacks the spatial resolution and sensitivity to structure and composition to provide insights on individual nanocrystals. Scanning transmission electron microscopy (STEM), is widely used to study the structure and composition of individual nanocrystals. It has recently been adapted with high sensitivity cathodoluminescence detectors that offer the opportunity to investigate the optical properties of individual nanostructures, an approach known as nano-cathodoluminescence (nano-CL) ${ }^{17}$. Nano-CL has been shown to reveal the effect of carrier recombination mechanisms on the emission of individual quantum structures ${ }^{18,19}$.

Here we perform nano-CL to reveal the effect of the structure and size of individual inorganic $\mathrm{CsPb}_{3}$ perovskite nanocrystals on their optical emission. We show that the nanocrystal size affects the emission dynamics caused by variations in the rate of trap defect and Auger recombination. In mixed solutions of $\mathrm{CsPbI}_{3}$ and $\mathrm{CsPbCl}_{3}$ nanocrystals, nano-CL reveals bright red and blue emission respectively from individual nanocrystals suitable for multi-wavelength emission with nanoscale uniformity ${ }^{20}$.

Nanocrystals of $\mathrm{CsPbI}_{3}$ were prepared using a solution based method previously reported by Protesecu et al. ${ }^{21}$, and deposited on a transparent support film. Bright red emission is observed from $\mathrm{CsPbI}_{3}$ solution under UV excitation $(365 \mathrm{~nm})$ at a concentration of $1 \mathrm{mg} / \mathrm{mL}$, as shown in Figure 1a. The photoluminescence (PL) spectrum of the solution (Figure 1b), shows emission centred at $1.79 \mathrm{eV}$ (694 nm) with a $100 \mathrm{meV}$ linewidth at the FWHM. 
Figure 1c shows the relative PL quantum efficiency $\left(Q E_{P L}\right)$ as a function of the photon fluence of the excitation laser pulse, $J_{p}$, for ensembles of $\mathrm{CsPbI}_{3}$ nanocrystals. Here $Q E_{P L}$ is defined as the ratio between the time-averaged, spectrally-integrated PL signal and the excitation fluence, normalised to the maximum value of this ratio. The $Q E_{P L}$ initially increases with photon fluence until reaching a plateau region before beginning to decrease again as the photon fluence is increased further.

At low fluence, the probability of absorbing more than one photon per nanocrystal per excitation pulse is negligible and so only single excitons are present in the sample. The $Q E_{P L}$ is then determined by the competition between radiative recombination and trap-mediated non-radiative channels. The increase of $Q E_{P L}$ with increasing excitation fluence corresponds to the gradual saturation of the trap states. The deactivation of the non-radiative recombination channels must have a lifetime similar to, or longer than, the interval between excitation pulses. Studies of luminescence intermittency have shown that suitably long-lived changes, $\sim 1 \mathrm{~s}$ in duration in some cases, to the emission properties of $\mathrm{CsPbI}_{3}$ nanocrystals are observed ${ }^{22}$.

The plateau region corresponds to fluences where the probability of absorbing more than one photon per nanocrystal becomes significant, and the increase of $Q E_{P L}$ due to trap saturation begins to be offset by Auger recombination. It has previously been shown that Auger recombination in perovskite nanocrystals has a characteristic lifetime that is much shorter than that of radiative recombination ${ }^{23}$ and so dominates when multi-excitons form, in trapping and trap-free nanocrystals alike. As the fluence increases further, the probability of more than one photon being absorbed per nanocrystal per pulse becomes greater and so an increasing number of nanocrystals contain multiple excitons which undergo Auger recombination. This causes a decrease in $Q E_{P L}$.

As described in the Supporting Information, fitting to the data in Figure 1c yielded an absorption cross-section of $\sigma_{a b s}=(2.9 \pm 0.2) \times 10^{-14} \mathrm{~cm}^{-2}$ at the excitation wavelength of $400 \mathrm{~nm}$ consistent with the value reported previously for $\mathrm{CsPbI}_{3}$ nanocrystals ${ }^{24}$; this cross-section was used to calculate 
the average number of photons absorbed per excitation pulse, $\langle N\rangle=\sigma_{a b s} J_{p}$, at each fluence, as also shown in Fig. 1c.

For greater insights into the relationship between emission properties of individual nanocrystals and nanocrystal structure, nano-CL was performed in a scanning transmission electron microscope (STEM). Miniature elliptical mirrors positioned around the specimen were used to collect the light emitted from each position in the sample as the sub-nanometre electron probe was rastered across the specimen, schematically shown in Figure 2a. The collected light was coupled to optical fibres and detected using a photomultiplier tube to give the CL intensity at each pixel. Simultaneously the inelastically scattered electrons were recorded on an annular dark field (ADF) detector and electron energy loss spectrometer (EELS) to reveal the structure and composition in addition to the optical emission.

ADF STEM images, such as the one shown in Figure $2 b$, show the morphology and sizes of the nanocrystals. The $\mathrm{CsPbI}_{3}$ nanocrystals are cuboidal in shape with an average size of $\sim 18 \mathrm{~nm}$ and a low aspect ratio (see Table 1 in SI). A histogram of their size distribution is shown in the supplementary information Figure S1. The degree of quantum confinement is given as the ratio of the Bohr radius $(a)$, which for $\mathrm{CsPbI}_{3}$ is $6 \mathrm{~nm}{ }^{21}$, to the length of the nanocrystals $(L / 2 a)$. This ratio is 1.5 , indicating intermediate confinement where the nanocrystal size is comparable to that of the bulk exciton.

The panchromatic CL image of the $\mathrm{CsPbI}_{3}$ nanocrystals (Figure 2c) shows CL that directly correlates with individual nanocrystals in the ADF-STEM image. Homogeneous emission is observed across the surface of each $\mathrm{CsPbI}_{3}$ nanocrystal. This is shown in Figure $\mathrm{S} 3$ for a different area of the specimen. The larger nanocrystals identified in the ADF-STEM image exhibit brighter CL, independent of their aspect ratio and shape.

Nano-CL offers the opportunity to study the effect of individual nanocrystal size on emission efficiency directly. To investigate the effect of nanocrystal volume on CL emission, we segmented the $\mathrm{CL}$ and ADF-STEM images to measure the area and thickness of more than 900 individual $\mathrm{CsPbI}_{3}$ nanocrystals (further details of the analysis approach can be found in SI, Figure S4). The probability of inelastic scattering of beam electrons by the specimen increases linearly with thickness over this 
range, and directly relates to the probability of generating excited carriers. The CL emission for each nanocrystal is averaged over the area and normalised to the thickness to yield CL per volume, so that the emission data is independent of excitation and only reflects recombination processes.

The colour scatter plot in Figure 3a shows the correlation between nanocrystal thickness, area and nanocrystal CL emission per volume. An increase in CL emission is observed with both thickness and area, supporting the qualitative effect apparent in Figure 2c. The CL is dominated by the largest and thickest nanocrystals, whilst the smallest nanocrystals exhibit the lowest radiative recombination intensity. Figure $3 \mathrm{~b}$ displays the scatter plot of the nanocrystal CL emission per volume against volume. The $\mathrm{CL}$ emission of the $\mathrm{CsPbI}_{3}$ increases super-linearly with the volume, and is described by a power law with an exponent of 1.2 .

The high electron energies used in STEM create multiple excitons in individual nanocrystals even from single interactions with beam electrons. This leads to large populations of excited carrier which then have a high probability of undergoing Auger recombination. Owing to the relatively long radiative lifetime of $\mathrm{CsPbI}_{3}$ nanocrystals ( $\left.\sim 40 \mathrm{~ns}\right)$ compared to the lifetime for Auger recombination ( $\left.\sim 100 \mathrm{ps}\right)$ ${ }^{16}$, radiative recombination is only likely to occur for the final exciton, for which Auger recombination is not possible. An increasing rate of Auger recombination relative to the rate of radiative recombination as the nanocrystal volume decreases, does not therefore on its own explain the reduced CL emission per volume observed for smaller nanocrystals.

The fluence dependence of $Q E_{P L}$ discussed above shows that trap-mediated recombination is an important recombination channel for these nanocrystals. The relative contribution of each nanocrystal to PL is determined by the size-dependence of the absorption cross-section, as discussed in detail in the SI. The trap density and trap-mediated recombination rate have been shown to increase with smaller size in other nanocrystals ${ }^{25}$, which results in greater competition with radiative recombination and thus decreased emission. Overcoming non-radiative recombination at trap states is therefore crucial to realise the benefits of semiconductor nanocrystals in optoelectronic devices. 
The development of mixtures of different discrete nanocrystal species, offers the potential for multiwavelength optoelectronic devices with nanoscale emission uniformity. We use nano-CL to study bright red and blue emission of self-assembled layers of $\mathrm{CsPbI}_{3}$ and $\mathrm{CsPbCl}_{3}$ nanocrystals respectively in a mixed solution.

A mixed solution was obtained by solution-mixing of the two nanocrystal species in an oxygen- and water-free environment ${ }^{26}$. The ADF-STEM image shown in Figure 4a reveals two distinct nanocrystal species corresponding to individual $\mathrm{CsPbI}_{3}$ and $\mathrm{CsPbCl}_{3}$ nanocrystal species. The $\mathrm{CsPbI}_{3}$ nanocrystals can be identified from their larger, more regular morphology and brighter contrast. These are surrounded by smaller, $\mathrm{CsPbCl}_{3}$ nanocrystals that show a broader distribution of sizes and shapes (size distribution and aspect ratios are given in SI Table 1 and Fig.S2). EELS compositional analysis confirms there are two discrete nanocrystal populations as shown in Figure 4b and in Figure S5.

Individual nanocrystals give rise to discrete emission as seen in the monochromatic CL images at $700 \mathrm{~nm}$ (shown in red) and $400 \mathrm{~nm}$ (shown in blue) in Figure 4c, corresponding to $\mathrm{CsPbI}_{3}$ and $\mathrm{CsPbCl}_{3}$ respectively. All $\mathrm{CsPbI}_{3}$ nanocrystals in the mixed specimen show uniform, bright $\mathrm{CL}$, while only the largest $\mathrm{CsPbCl}_{3}$ nanocrystals show efficient emission.

The CL emission spectrum averaged over all the nanocrystals in Figure 4 shows two emission peaks at $700 \mathrm{~nm}$ and $400 \mathrm{~nm}$. No other emission wavelengths are detected, which would typically be associated with the red-shifted orthorhombic phase, reflecting the high crystal quality of the inorganic perovskite nanocrystals in this mixed solution.

We studied the luminescence of individual inorganic perovskite nanocrystals, to reveal how it depends on size and recombination mechanisms. The fine spatial resolution of nano-CL shows the relationship between nanoscale optical emission and size of individual nanocrystals. This approach reveals that $\mathrm{CL}$ emission per volume is greater in larger nanocrystals, which we attribute to reduced trap-mediated recombination. Developing strategies to suppress the effects of surface trap states, such as engineering the nanocrystal size and employing heterostructures ${ }^{27,28}$ will be critical to developing higher efficiency light emission and harvesting devices. Nano-CL identified bright red and blue 
emission from discrete $\mathrm{CsPbI}_{3}$ and $\mathrm{CsPbCl}_{3}$ nanocrystals respectively in mixed solutions. This offers multi-wavelength emission with nanoscale colour uniformity. The insights gained by the combination of optical and structural techniques have aided in the fundamental understanding of colloidal semiconductor nanocrystals and will support the development of future optoelectronic devices.

\section{Acknowledgements}

F.W.R.R. , F.d.P.M, and C.D. acknowledge funding from the ERC under grant number 259619 PHOTO EM. This work was supported by EPSRC (EP/P02484X/1). N.J.L.K.D. thanks the Ernest Oppenhimer Trust for a research fellowship. R. A-L. thanks CONACYT for provision of a scholarship (284566/399936).

\section{ALL FUNDING SOURCES Please}

\section{Materials and methods}

\section{Perovskite synthesis}

Perovskite nanocrystals were synthesized using previously reported procedures ${ }^{21}$. $\mathrm{Cs}_{2} \mathrm{CO}_{3}(0.814 \mathrm{~g}$, 99.9\%) was loaded into $100 \mathrm{~mL}$ three-neck flask along with octadecene (ODE, $30 \mathrm{~mL}, 90 \%)$ and oleic acid $(2.5 \mathrm{~mL}, \mathrm{OA}, 90 \%)$, the mixture was dried for $2 \mathrm{~h}$ at $120^{\circ} \mathrm{C}$ under $\mathrm{N}_{2}$. The solution temperature was then lowered to $100^{\circ} \mathrm{C}$. ODE $(75 \mathrm{~mL})$, oleylamine $(7.5 \mathrm{~mL}$, OLA, $90 \%)$, and dried $\mathrm{OA}(7.5 \mathrm{~mL})$ and $\mathrm{PbX}_{2}(2.82 \mathrm{mmol})$ such as $\mathrm{PbI}_{2}(1.26 \mathrm{~g}, 99.99 \%), \mathrm{PbCl}_{2}(0.675 \mathrm{~g}, 99.99 \%)$, were loaded into a 250 $\mathrm{mL}$ three-neck flask and dried under vacuum for $2 \mathrm{~h}$ at $120^{\circ} \mathrm{C}$. After complete solubilisation of the $\mathrm{PbX} \mathrm{X}_{2}$ salt, the temperature was raised to $170^{\circ} \mathrm{C}$ and the Cs-oleate solution $(6.0 \mathrm{~mL}, 0.125 \mathrm{M}$ in ODE, prepared as described above) was quickly injected. After $10 \mathrm{~s}$, the reaction mixture was cooled in an ice-water bath. For $\mathrm{CsPCl}_{3}$ synthesis, $5 \mathrm{~mL}$ of trioctylphosphine (TOP, 97\%) was added to solubilize $\mathrm{PbCl}_{2}$. The nanocrystals were transferred to an argon gloved box $\left(\mathrm{H}_{2} \mathrm{O}\right.$ and $\left.\mathrm{O}_{2}<1 \mathrm{ppm}\right)$ precipitated from solution by the addition of equal volume anhydrous butanol ( $\mathrm{BuOH}, 99 \%)(\mathrm{ODE}: \mathrm{BuOH}=1: 1$ by volume). After centrifugation, the supernatant was discarded and the nanocrystals were redispersed in anhydrous 
hexane (99\%) and precipitated again with the addition of $\mathrm{BuOH}$ (hexane:BuOH $=1: 1$ by volume). These were redispersed in hexane. The nanocrystal dispersion was filtered through a $0.2 \mu \mathrm{m}$ PTFE filter and diluted to $50 \mathrm{mg} / \mathrm{mL}$ in hexane before use. Specimens for characterisation were prepared and stored in the glove box to minimise exposure to air and moisture.

\section{Scanning transmission electron microscopy}

Samples for STEM were prepared by drop-casting $40 \mathrm{mg} / \mathrm{mL}$ perovskite nanocrystal solution in hexane on a carbon coated 200 mesh copper grid in an argon filled glove box. Specimens were transported in inert atmosphere and only exposed to the environment briefly when loaded onto the TEM specimen holder. A liquid nitrogen holder was used to cool the specimen to $100 \mathrm{~K}$. Nano-CL measurements were performed on a Jeol $2100 \mathrm{~F}$ operating at $80 \mathrm{keV}$ with $6 \mathrm{pA}$ fitted with a Gatan Vulcan CL system. Typical dwell times for imaging at intermediate magnifications were $16 \mu$ s/pixel (dose $\sim 15 \mathrm{e}^{-} / \AA^{2}$ ), and for spectroscopy were $100 \mu$ s (dose $\sim 85 \mathrm{e}^{\mathrm{e}} / \AA^{2}$ ). Miniature elliptical mirrors around the specimen were used to collect the light and then coupled to optical fibres before being detected on a photomultiplier tube. EELS measurements were recorded on a Gatan Quantum SE spectrometer.

\section{Photoluminescence measurements}

PL spectra were measured on an Edinburgh instruments FLS980 fluorimeter in solution in a $10 \mathrm{~mm}$ $\times 3 \mathrm{~mm}$ cuvette excited along the longest side and recorded along the shortest side.

Ensembles of the nanocrystals were studied by fluence-dependent photoluminescence (PL) spectroscopy. This was performed using a pulsed Ti:sapphire (RegA 9000) laser source at a repetition rate of $250 \mathrm{kHz}$ and $170 \mathrm{fs}$ pulse duration. A frequency doubler (PHOTOP TP-2000B) was used to set the excitation wavelength at $400 \mathrm{~nm}$. The laser fluence was varied with a neutral density filter wheel and measured by a power detector. The spot size at the sample position was measured to be $1.3 \times 10^{-3}$ $\mathrm{mm}^{2}$ after defocusing with an objective lens. The emitted light was collected by an optic fibre and directed into a spectrometer (Ocean Optics) after passing through a long pass filter to eliminate scattered light from the source. More details of this experimental setup are given in Reference ${ }^{29}$. 
(1) Alivisatos, A. P.; Peng, X.; Manna, L.; Yang, W.; Wickham, J.; Scher, E.; Kadavanich, A. Shape control of CdSe nanocrystals. Nature 2000, 404, 59-61.

(2) Burda, C.; Chen, X.; Narayanan, R.; El-Sayed, M. A. Chemistry and properties of nanocrystals of different shapes. Chemistry and Properties of Nanocrystals of Different Shapes. Chemical Reviews, 2005, 105, 1025-1102.

(3) Shirasaki, Y.; Supran, G. J.; Bawendi, M. G.; Bulović, V. Emergence of colloidal quantumdot light-emitting technologies. Nat. Photonics 2012, 7, 13-23.

(4) Dai, Q.; Duty, C. E.; Hu, M. Z. Semiconductor-Nanocrystals-Based White Light-Emitting Diodes. Small 2010, 6, 1577-1588.

(5) Klimov, V. I.; Mikhailovsky, A. A.; Xu, S.; Malko, A.; Hollingsworth, J. A.; Leatherdale, C. A.; Eisler, H.; Bawendi, M. G. Optical Gain and Stimulated Emission in Nanocrystal Quantum Dots. Science 2000, 290, 314-317.

(6) Yuan, M.; Liu, M.; Sargent, E. H. Colloidal quantum dot solids for solution-processed solar cells. Nat. Energy 2016, 1, 16016.

(7) Wang, X.; Zhuang, J.; Peng, Q.; Li, Y. A general strategy for nanocrystal synthesis. Nature 2005, 437, 121-124.

(8) Stranks, S. D.; Snaith, H. J. Metal-halide perovskites for photovoltaic and light-emitting devices. Nat. Nanotechnol. 2015, 10,391-402.

(9) Hong, K.; Le, Q. Van; Kim, S. Y.; Jang, H. W. J. Low-dimensional halide perovskites: review and issues. Mater. Chem. C 2018, 6, 2189-2209.

(10) Efros, A. L.; Nesbitt, D. J. Origin and control of blinking in quantum dots. Nat. Nanotechnol. $2016,11,661-671$.

(11) Califano, M.; Gómez-Campos, F. M. Universal Trapping Mechanism in Semiconductor Nanocrystals. Nano Lett. 2013, 13, 2047-2052.

(12) Califano, M. Origins of Photoluminescence Decay Kinetics in CdTe Colloidal Quantum Dots. ACS Nano 2015, 9, 2960-2967.

(13) Sher, P. H.; Smith, J. M.; Dalgarno, P. A.; Warburton, R. J.; Chen, X.; Dobson, P. J.; Daniels, S. M.; Pickett, N. L.; O’Brien, P. Power law carrier dynamics in semiconductor nanocrystals at nanosecond timescales. Appl. Phys. Lett. 2008, 92, 101111.

(14) Vaxenburg, R.; Rodina, A.; Lifshitz, E.; Efros, A. L. Biexciton Auger Recombination in CdSe/CdS Core/Shell Semiconductor Nanocrystals. Nano Lett. 2016, 16, 2503-2511. 
(15) Robel, I.; Gresback, R.; Kortshagen, U.; Schaller, R. D.; Klimov, V. I. Universal SizeDependent Trend in Auger Recombination in Direct-Gap and Indirect-Gap Semiconductor Nanocrystals Phys. Rev. Lett. 2009, 102, 177404.

(16) Makarov, N. S.; Guo, S.; Isaienko, O.; Liu, W.; Robel, I.; Klimov, V. I. Spectral and Dynamical Properties of Single Excitons, Biexcitons, and Trions in Cesium-Lead-Halide Perovskite Quantum Dots. Nano Lett. 2016, 16, 2349-2362.

(17) Kociak, M.; Zagonel, L. F. Cathodoluminescence in the scanning transmission electron microscope. Ultramicroscopy 2017, 176, 112-131.

(18) Zagonel, L. F.; Tizei, L. H. G.; Vitiello, G. Z.; Jacopin, G.; Rigutti, L.; Tchernycheva, M.; Julien, F. H.; Songmuang, R.; Ostasevicius, T.; de la Peña, F.; Ducati, C.; Midgley, P. A.; Kociak, M. Nanometer-scale monitoring of quantum-confined Stark effect and emission efficiency droop in multiple GaN/AlN quantum disks in nanowires. Phys. Rev. B 2016, 93, 205410.

(19) Griffiths, J. T.; Zhang, S.; Rouet-Leduc, B.; Fu, W. Y.; Bao, A.; Zhu, D.; Wallis, D. J.; Howkins, A.; Boyd, I.; Stowe, D.; Kappers, M. J.; Humphreys, C. J.; Oliver, R. A.

Nanocathodoluminescence Reveals Mitigation of the Stark Shift in InGaN Quantum Wells by Si Doping. Nano Lett. 2015, 15, 7639-7643.

(20) Davis, N. J. L. K.; de la Peña, F. J.; Tabachnyk, M.; Richter, J. M.; Lamboll, R. D.; Booker, E. P.; Wisnivesky Rocca Rivarola, F.; Griffiths, J. T.; Ducati, C.; Menke, S. M.; Deschler, F.; Greenham, N. C. Photon Reabsorption in Mixed $\mathrm{CsPbCl}_{3}: \mathrm{CsPbI}_{3}$ Perovskite Nanocrystal Films for LightEmitting Diodes. J. Phys. Chem. C 2017, 121, 3790-3796.

(21) Protesescu, L.; Yakunin, S.; Bodnarchuk, M. I.; Krieg, F.; Caputo, R.; Hendon, C. H.; Yang, R. X.; Walsh, A.; Kovalenko, M. V. Nanocrystals of Cesium Lead Halide Perovskites $\left(\mathrm{CsPbX}_{3}, \mathrm{X}=\right.$ $\mathrm{Cl}, \mathrm{Br}$, and I): Novel Optoelectronic Materials Showing Bright Emission with Wide Color Gamut. Nano Lett. 2015, 15, 3692-3696.

(22) Park, Y.-S.; Guo, S.; Makarov, N. S.; Klimov, V. I. Room Temperature Single-Photon Emission from Individual Perovskite Quantum Dots. ACS Nano 2015, 9, 10386-10393.

(23) Klimov, V. I. Multicarrier Interactions in Semiconductor Nanocrystals in Relation to the Phenomena of Auger Recombination and Carrier Multiplication. Annu. Rev. Condens. Matter Phys. 2014, 5, 285-316.

(24) Makarov, N. S.; Guo, S.; Isaienko, O.; Liu, W.; Robel, I.; Klimov, V. I. Spectral and Dynamical Properties of Single Excitons, Biexcitons, and Trions in Cesium-Lead-Halide Perovskite Quantum Dots. Nano Lett. 2016, 16, 2349-2362. 
(25) Bozyigit, D.; Lin, W. M. M.; Yazdani, N.; Yarema, O.; Wood, V. A quantitative model for charge carrier transport, trapping and recombination in nanocrystal-based solar cells. Nat.

Commun. 2015, 6, 6180.

(26) Davis, N. J. L. K.; Peña, F. J. de la; Tabachnyk, M.; Richter, J. M.; Lamboll, R. D.; Booker, E. P.; Rivarola, F. W. R.; Griffiths, J. T.; Ducati, C.; Menke, S. M.; Deschler, F.; Greenham, N. C. Photon Reabsorption in Mixed CsPbCl3:CsPbI3 Perovskite Nanocrystal Films for Light-Emitting Diodes J. Phys. Chem. C 2017, 121, 3790-3796. Hollingsworth, J. A. Heterostructuring Nanocrystal Quantum Dots Toward Intentional Suppression of Blinking and Auger Recombination. Chem. Mater. 2013, 25, 1318-1331.

(28) Zavelani-Rossi, M.; Lupo, M. G.; Tassone, F.; Manna, L.; Lanzani, G. Suppression of Biexciton Auger Recombination in CdSe/CdS Dot/Rods: Role of the Electronic Structure in the Carrier Dynamics. Nano Lett. 2010, 10, 3142-3150.

(29) Alanis, J. A.; Saxena, D.; Mokkapati, S.; Jiang, N.; Peng, K.; Tang, X.; Fu, L.; Tan, H. H.; Jagadish, C.; Parkinson, P. Large-Scale Statistics for Threshold Optimization of Optically Pumped Nanowire Lasers. Nano Lett. 2017, 17, 4860-4865.

Figure 1: (a) The optical emission from the CsPbI 3 nanocrystal solution under UV illumination (b) PL emission spectra from $\mathrm{CsPbI}_{3}$ nanocrystals. (c) Normalised photoluminescence quantum efficiency, $Q E_{\mathrm{PL}}$, as function of photon fluence, $J_{p}$, and average number of photons absorbed per pulse, $\langle N\rangle$.

Figure 2: a) Schematic diagram of the STEM fitted with a CL system, showing the trajectory of the electrons in green and the collection of the emitted CL in yellow. (b) ADF-STEM image of the CsPbI nanocrystals reveals the structure. (c) The panchromatic $\mathrm{CL}$ image of $\mathrm{CsPbI}_{3}$ shows bright luminescence that corresponds with each of the nanocrystals in the corresponding ADF-STEM image. The total width of all images is $350 \mathrm{~nm}$.

Figure 3: (a) Colour scatter plot reveal the individual impact of the nanocrystal area and thickness on the normalised $C L$ emission per volume shown in colour. (b) The effect of the nanocrystal volume on the $C L$ emission per volume. The solids line is a power law fit with the index a fitting parameter.

Figure 4: a) ADF-STEM image of the mixed $\mathrm{CsPbI}_{3}$ and $\mathrm{CsPbCl}_{3}$ nanocrystals. b) EELS of the regions highlighted by the white box on the ADF-STEM image shows the halogen composition of the nanocrystals. c) The corresponding monochromatic CL images at $700 \mathrm{~nm}$ and $400 \mathrm{~nm}$ reveal distinct emission arising from $\mathrm{CsPbI}_{3}$ and $\mathrm{CsPCCl}_{3}$ in the corresponding ADF-STEM image. d) The averaged CL emission reveals the emission from $\mathrm{CsPbI}_{3}$ at $700 \mathrm{~nm}$ and emission from the $\mathrm{CsPbCl}_{3}$ at $400 \mathrm{~nm}$. 